\title{
Exploiting lipotoxicity for the treatment of liver cancer
}

\author{
Ramona Rudalska (iD) ${ }^{1}$, Lars Zender ${ }^{1,2,3}$ and Daniel Dauch (iD ${ }^{1,2,3}{ }^{凶}$
}

(c) The Author(s) 2021

Metabolic alterations occur frequently in solid tumours, but metabolic cancer therapies are limited by the complexity and plasticity of metabolic networks. We could recently show that activation of the liver X receptor alpha (LXRa) and inhibition of a Raf-1-SCD1 protein complex induces an intracellular accumulation of saturated free fatty acids leading to lethal lipotoxicity in tumour cells and allows for an efficient treatment of liver carcinomas.

British Journal of Cancer (2021) 125:1459-1461; https://doi.org/10.1038/s41416-021-01479-7

Solid tumours frequently accumulate alterations in metabolic pathways during development [1, 2]. A metabolic reprogramming may fuel tumour growth due to the specific biosynthetic demands of cancer cells e.g. an increased requirement for lipids for membrane biosynthesis [3]. Furthermore, cancer cells may need to adapt their metabolism to a specific tumour microenvironment and hypoxia [1,2]. Since metabolic reprogramming in tumour cells may induce dependencies on specific metabolic cascades, therapeutic concepts that exploit metabolic vulnerabilities of tumors have gained major interest in cancer research [1, 2]. Especially, enzymes involved in the de novo synthesis of lipids and their precursors such as fatty acid synthase (FASN), stearoyl-CoA desaturase 1 (SCD1), ATP-citrate lyase (ACLY) and acyl-CoA synthetase short chain family member 2 (ACSS2) represent potential targets for therapeutic approaches [3]. A pharmacological FASN inhibitor (TVB-2640) is currently being tested in a phase II clinical trial against KRAS mutated Non-Small Cell Lung Carcinomas (NCT03808558).

However, the development of cancer therapies that are based on the inhibition of important biosynthetic pathways is often impaired by the diversity and plasticity of metabolic networks [1-3]. As a consequence, only a subfraction of tumours may respond to such therapies and a patient selection might be required prior to treatment onset. Furthermore, acquired therapy resistance can rapidly evolve and limit metabolic therapies by activation of compensatory pathways or by an intensified exchange of metabolites between cancer cells and the tumour environment $[1,3]$.

Notably, metabolic adaptations may render tumour cells also sensitive to toxic metabolites such as reactive oxygen species, trans- or saturated fatty acids [4-6]. A therapy that exploits an intracellular accumulation of toxic metabolites may be specific to tumour cells and independent of the individual tumour metabolism. Therefore, more patients may benefit from such a therapeutic approach.
We could recently show that activation of de novo fatty acid synthesis in combination with an inhibition of fatty acid desaturation results in an accumulation of saturated fatty acids in hepatocellular carcinoma (HCC) cells. Such an accumulation of toxic saturated fatty acids triggers severe lipotoxicity and apoptosis in cancer cells and allows for an efficient treatment of liver tumours with different genetic and metabolic makeups [7].

In order to induce de novo fatty acid synthesis, we exposed HCC cells to synthetic activators of the liver $X$ receptor alpha (LXRa). $L X R a$ forms a heterodimer with retinoid $X$ receptor (RXR) on the LXR response element and controls the expression of genes involved in fatty acid and cholesterol metabolism [8, 9]. The LXRRXR heterodimer exists either in a basal state where its transcriptional activity is restricted by co-repressors, or in an active state, which is triggered by natural (oxysterols) or synthetic (T0901317, GW3965) ligands and results in the binding of coactivators $[8,9]$. Upon activation, LXRa induces expression of sterol regulatory element binding transcription factor 1 (SREBF1), acetylCoA carboxylase alpha (ACACA) and FASN, which in turn produces C16:0 and C18:0 fatty acids in presence of their metabolic substrates [10].

These substrates, such as Acetyl-CoA, ATP and NADPH, are often abundant in tumour cells [3]. However, a pharmacological activation of LXRa alone does not lead to lipotoxicity and cell death in cancer cells, since saturated $\mathrm{C} 16 / \mathrm{C} 18$ fatty acids are rapidly desaturated by SCD1 (another downstream target of LXRa [10]) to nontoxic mono-unsaturated fatty acids (Fig. 1a). Surprisingly, we identified the RAF proto-oncogene serine/threonineprotein kinase (Raf-1) as a critical activator of SCD1 in HCC cells. We found that Raf-1, an important member of the Ras/MAPK pathway, directly interacts with the desaturase and delays the turnover of SCD1 [7], a short-lived protein with a half-life of 2 to 4 hours [11]. We assume that this protein-protein interaction increases the capability of cancer cells to desaturate toxic

\footnotetext{
${ }^{1}$ Department of Medical Oncology and Pneumology, University Hospital Tuebingen, Tuebingen, Germany. ${ }^{2}$ German Cancer Research Consortium (DKTK), German Cancer Research Center (DKFZ), Heidelberg, Germany. ${ }^{3}$ iFIT Cluster of Excellence EXC 2180 "Image-Guided and Functionally Instructed Tumor Therapies", University of Tuebingen, Tuebingen, Germany. ${ }^{凶}$ email: daniel.dauch@med.uni-tuebingen.de
}

Received: 7 April 2021 Revised: 11 June 2021 Accepted: 30 June 2021

Published online: 23 July 2021 
a

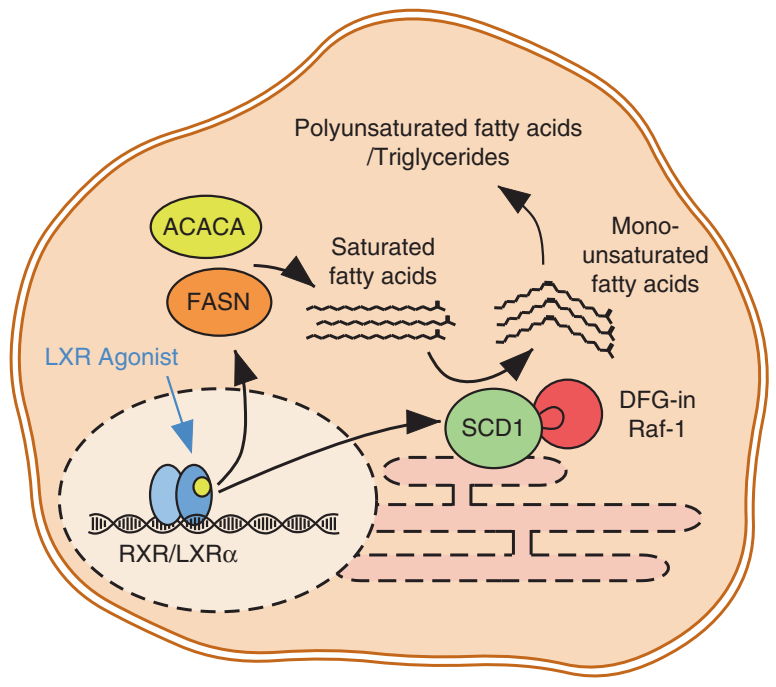

b

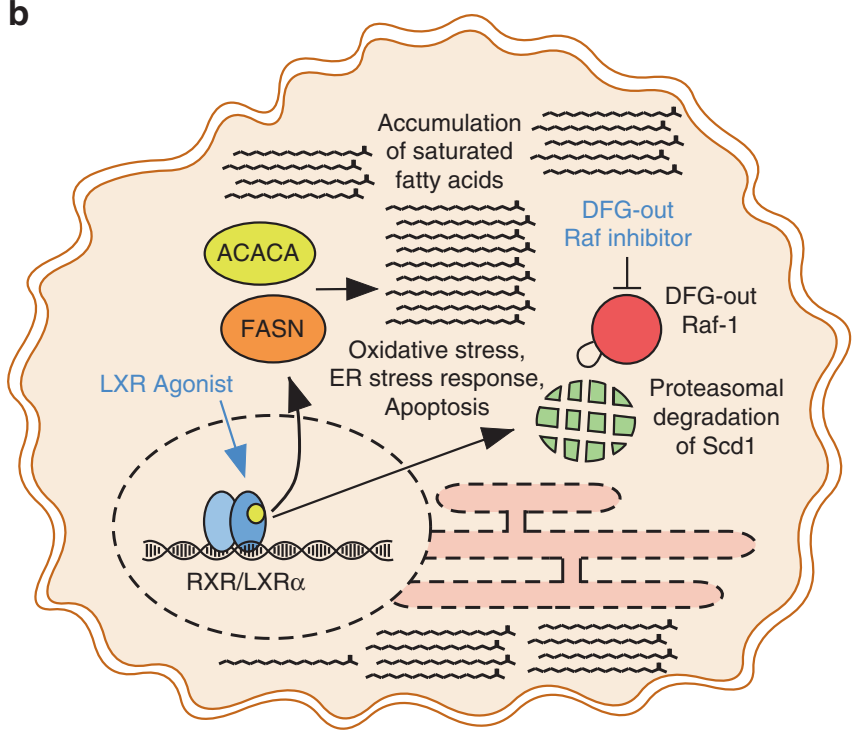

Fig. 1 Induction of lipotoxicity for the treatment of liver cancer. a Activation of LXR $\alpha$ alone induces de novo fatty acid synthesis in cancer cells. However, saturated fatty acids are rapidly desaturated by SCD1 due to a Raf-1 mediated stabilization of the enzyme. $\mathbf{b}$ Inhibition of Raf- 1 by DFG-out Raf inhibitors induces proteasomal degradation of SCD1 leading to an intracellular accumulation of toxic saturated fatty acids, lipotoxicity and consequently to apoptosis of liver cancer cells upon LXR $\alpha$ activation.

saturated fatty acids, highlighting Raf-1 as an important regulator of lipid metabolism in cancer (Fig. 1a).

In order to target the Raf-1-SCD1 protein complex in HCC, we treated liver cancer cells with different Raf inhibitors. While compounds that induce an "in" conformation of the DFG motif in the kinase (e.g. dabrafenib, SB590885) [12] were not able to affect the Raf-1-SCD1 interaction, DFG-out Raf inhibitors such as sorafenib or BI-882370 [12] efficiently disrupt this protein complex leading to rapid proteasomal degradation of SCD1 (Fig. 1b). In line with these data, a combinatorial therapy comprising an LXRa agonist and a DFG-out Raf inhibitor triggers a strong intracellular accumulation of saturated fatty acids in liver cancer cells. Such an accumulation of toxic lipids results in oxidative stress, a critical ER stress response (activation of PERK and its downstream targets elF2a, GADD34 and (HOP), a formation of lipid droplets and subsequently in apoptosis of liver cancer cells [7] (Fig. 1b). Treatment studies in autochthonous, transposon-based liver cancer mouse models [13] and xenograft models of human HCC revealed, that this combinatorial therapy is highly effective in order to suppress genetically and metabolically different liver carcinomas and to significantly extend the survival of tumour bearing animals.

HCC represents a leading cause of cancer-related death worldwide and it is expected that the HCC incidence will further rise in the future due to increasing rates of fatty liver diseases such as non-alcoholic steatohepatitis (NASH) [14]. HCCs exhibit strong resistance to cytotoxic and targeted therapies [14, 15] and only a subtype of tumours responds to recently developed immunotherapies [16]. Especially, the increasing numbers of NASH-associated HCCs are largely resistant to checkpoint inhibitors [16, 17]. Importantly, our novel lipotoxic therapy was effective against NASH-driven HCCs, indicating a strong potential for the treatment of NASH-HCC patients in the future. Since this therapy was well tolerated by mice, even by animals that suffer from severe NASH [7], we believe that a therapeutic window for the treatment of human liver cancer patients exits.

The DFG-out Raf inhibitor sorafenib is routinely used for the treatment of liver cancer patients $[15,18]$ and could be therefore easily applied as a component of our combinatorial lipotoxic therapy. Since LXRa agonists are not yet approved for the treatment of patients, the development of potent and specific LXRa agonists might be an important task for the future in order to translate our findings into the clinic.

\section{DATA AVAILABILITY}

Not applicable.

\section{REFERENCES}

1. Schulze A, Harris AL. How cancer metabolism is tuned for proliferation and vulnerable to disruption. Nature. 2012;491:364-73.

2. Luengo A, Gui DY, Vander Heiden MG. Targeting metabolism for cancer therapy. Cell Chem Biol. 2017;24:1161-80.

3. Koundouros N, Poulogiannis G. Reprogramming of fatty acid metabolism in cancer. Br J Cancer. 2020;122:4-22.

4. Reczek CR, Chandel NS. The two faces of reactive oxygen species in cancer. Annu Rev Canc Biol. 2017;1:79-98.

5. Williams KJ, Argus JP, Zhu Y, Wilks MQ, Marbois BN, York AG, et al. An essential requirement for the SCAP/SREBP signaling axis to protect cancer cells from lipotoxicity. Cancer Res. 2013;73:2850-62.

6. Munir R, Lisec J, Swinnen JV, Zaidi N. Lipid metabolism in cancer cells under metabolic stress. Br J Cancer. 2019;120:1090-8.

7. Rudalska R, Harbig J, Snaebjornsson MT, Klotz S, Zwirner S, Taranets L, et al. LXRa activation and Raf inhibition trigger lethal lipotoxicity in liver cancer. Nature Cancer. 2021;2:201-17.

8. Peet DJ, Janowski BA, Mangelsdorf DJ. The LXRs: a new class of oxysterol receptors. Curr Opin Genet Dev. 1998;8:571-5.

9. Hong C, Tontonoz P. Liver X receptors in lipid metabolism: opportunities for drug discovery. Nat Rev Drug Discov. 2014;13:433-44.

10. Schultz JR, Tu H, Luk A, Repa JJ, Medina JC, Li L, et al. Role of LXRs in control of lipogenesis. Genes Dev. 2000;14:2831-8.

11. Kato $H$, Sakaki $K$, Mihara K. Ubiquitin-proteasome-dependent degradation of mammalian ER stearoyl-CoA desaturase. J Cell Sci. 2006;119:2342-53.

12. Agianian B, Gavathiotis E. Current Insights of BRAF Inhibitors in Cancer. J Med Chem. 2018;61:5775-93.

13. Rudalska R, Dauch D, Longerich T, McJunkin K, Wuestefeld T, Kang TW, et al. In vivo RNAi screening identifies a mechanism of sorafenib resistance in liver cancer. Nat. Med. 2014;20:1138-46.

14. Llovet JM, Zucman-Rossi J, Pikarsky E, Sangro B, Schwartz M, Sherman M, et al. Hepatocellular carcinoma. Nat Rev Dis Primers. 2016;2:16018.

15. Llovet JM, Montal R, Sia D, Finn RS. Molecular therapies and precision medicine for hepatocellular carcinoma. Nat Rev Clin Oncol. 2018;15:599-616.

16. Pinter M, Scheiner B, Peck-Radosavljevic M. Immunotherapy for advanced hepatocellular carcinoma: a focus on special subgroups. Gut. 2021;70:204-14. 
17. Pfister D, Núñez NG, Pinyol R, Govaere O, Pinter M, Szydlowska M, et al. NASH limits anti-tumour surveillance in immunotherapy-treated HCC. Nature. 2021;592:450-6.

18. Wilhelm S, Carter C, Lynch M, Lowinger T, Dumas J, Smith RA, et al. Discovery and development of sorafenib: a multikinase inhibitor for treating cancer. Nat. Rev. Drug Discov. 2006;5:835-44.

\section{ACKNOWLEDGEMENTS}

Not applicable.

\section{AUTHOR CONTRIBUTIONS}

All authors wrote the manuscript.

\section{FUNDING INFORMATION}

This work was supported by the Deutsche Forschungsgemeinschaft (DFG, German Research Foundation) [FOR2314 (project number 267467939) (DD and LZ), SFB-TR209 - 314905040 (DD and LZ), SFB-TR240 - 374031971 (LZ), Gottfried Wilhelm Leibniz Program (LZ)], the Deutsche Forschungsgemeinschaft (DFG, German Research Foundation) under Germany's excellence strategy - EXC 2180 - 390900677 ["Image-Guided and Functionally Instructed Tumor Therapies" (iFIT) (LZ and DD)], the Landesstiftung Baden-Wuerttemberg ['Improve CRC' (DD and LZ)] and the German Cancer Research Consortium (DKTK) (LZ). Open Access funding enabled and organized by Projekt DEAL.

\section{ETHICS APPROVAL AND CONSENT TO PARTICIPATE}

Not applicable.

\section{CONSENT TO PUBLISH}

Not applicable.

\section{COMPETING INTERESTS}

The authors declare no competing interests.

\section{ADDITIONAL INFORMATION}

Correspondence and requests for materials should be addressed to D.D.

Reprints and permission information is available at http://www.nature.com/ reprints

Publisher's note Springer Nature remains neutral with regard to jurisdictional claims in published maps and institutional affiliations.

\section{cc) (1)}

Open Access This article is licensed under a Creative Commons Attribution 4.0 International License, which permits use, sharing, adaptation, distribution and reproduction in any medium or format, as long as you give appropriate credit to the original author(s) and the source, provide a link to the Creative Commons license, and indicate if changes were made. The images or other third party material in this article are included in the article's Creative Commons license, unless indicated otherwise in a credit line to the material. If material is not included in the article's Creative Commons license and your intended use is not permitted by statutory regulation or exceeds the permitted use, you will need to obtain permission directly from the copyright holder. To view a copy of this license, visit http://creativecommons. org/licenses/by/4.0/.

(c) The Author(s) 202 